\title{
VARIACIÓN ENTRE POBLACIONES DE Abies religiosa (H.B.K.) Schl. et Cham A LO LARGO DE UN GRADIENTE ALTITUDINAL. I. CAPACIDAD GERMINATIVA DE LA SEMILLA
}

\author{
VARIATION AMONG Abies religiosa (H.B.K.) Schl. et Cham POPULATIONS \\ ALONG AN ALTITUDINAL GRADIENT. I. SEED GERMINATION CAPACITY
}

\author{
Marisol A. Ortiz-Bibian 1, 2, Dante Castellanos-Acuña ${ }^{3,4}$, Mariela Gómez-Romero ${ }^{1,5}$ \\ Roberto Lindig-Cisneros ${ }^{6}$, Miguel Ángel Silva-Farías ${ }^{7}$ y Cuauhtémoc Sáenz-Romero ${ }^{8 *}$
}

\begin{abstract}
'Universidad Michoacana de San Nicolás de Hidalgo (UMSNH), Facultad de Biología, Morelia, Michoacán, México. ${ }^{2}$ Tecnológico de Monterrey, Departamento de Ciencias, Morelia, Michoacán, México. ${ }^{3} \mathrm{UMSNH}$, Instituto de Investigaciones Agropecuarias y Forestales, Morelia, Michoacán, México. ${ }^{4}$ University of Alberta, Department of Renewable Resources, Edmonton, Canadá. ${ }^{5}$ Cátedras CONACyT. ${ }^{6}$ Universidad Nacional Autónoma de México (IIES-UNAM), Instituto de Investigaciones en Ecosistemas y Sustentabilidad. Morelia, Michoacán, México. ${ }^{7}$ Servi-Ambiental El Bosque S.A. de C.V., Morelia, Michoacán. ${ }^{8} \mathrm{UMSNH}$, Instituto de Investigaciones sobre los Recursos Naturales, Morelia, Michoacán, México.
\end{abstract}

*Autor de correspondencia (csaenzromero@gmail.com)

\section{RESUMEN}

Abies religiosa (HBK) Schl. \& Cham. (oyamel) es una conífera nativa de México. El bosque de oyamel que se ubica en la frontera del Estado de México y Michoacán funge como refugio de poblaciones de mariposa monarca Danaus plexippus L., pero existe preocupación por el futuro de Abies religiosa en la región debido al cambio de uso de suelo y al cambio climático, pues se espera una reducción de su nicho ecológico. Con la finalidad de generar información que permita planear la cantidad de semilla necesaria a recolectar para realizar programas de reforestación a gran escala en la región de la Reserva de la Biósfera de la Mariposa Monarca, se evaluó la viabilidad y germinación de semilla de oyamel recolectada en 15 poblaciones a lo largo de un gradiente altitudinal ( 2850 a $3550 \mathrm{msnm}$, una cada $50 \mathrm{~m}$ ) en el cerro de San Andrés, Michoacán. Hubo diferencias estadísticamente significativas entre poblaciones en viabilidad $(P=0.0007)$ y germinación $(P<0.0001)$ de las semillas. Se encontró una relación cuadrática significativa de la viabilidad $\left(R^{2}\right.$ $=0.510, P=0.0138)$ y la germinación $\left(R^{2}=0.768, P=0.0002\right)$ con la altitud de origen de la procedencia. La semilla de poblaciones que se distribuyen entre 3000 y $3350 \mathrm{msnm}$ (parte media de la distribución altitudinal) posee el valor más alto de viabilidad (48.7 \%) y de germinación (19.6 \%), mayor al de los extremos altitudinales superior e inferior. Si se recolecta de la zona de altitud intermedia (Zona II, de 3000 a $3350 \mathrm{msnm}$ ) se requerirá recolectar aproximadamente $2.0 \mathrm{~kg}$ de semilla para producir 10,000 plantas en vivero; en cambio, la misma meta requeriría de cinco veces más semilla de la zona de menor altitud $(11.1 \mathrm{~kg}$, Zona I, 2650 a $3000 \mathrm{msnm})$. Esto indica las dificultades prácticas de abastecimiento de semilla a las que se enfrentaría un programa de reforestación a gran escala, especialmente si se requiere de germoplasma originado de las poblaciones de baja altitud, para implementar migración asistida hacia mayores altitudes para aminorar los efectos del cambio climático.

Palabras clave: Abies religiosa, cambio climático, germinación, gradiente altitudinal, migración asistida, viabilidad.

\section{SUMMARY}

Abies religiosa (HBK) Schl. \& Cham. (oyamel fir) is a conifer native to Mexico. The oyamel forest located on the border of the State of Mexico and Michoacán serves as a refuge for Monarch butterfly populations Danaus plexippus L., but there is concern about the future of Abies religiosa in the region due to the change in land use and the climate change; thus, a reduction of its ecological niche is expected. In order to generate information that allows plannning the amount of seed necessary to be collected to carry out large- scale reforestation programs in the region of the Monarch Butterfly Biosphere Reserve, the viability and germination of oyamel fir seeds collected from 15 populations along an altitudinal gradient (2850 to $3550 \mathrm{~m}$, one every $50 \mathrm{~m}$ ), on the San Andres hill, Michoacán, were evaluated. There were statistically significant differences among populations in seed viability $(P=0.0007)$ and germination $(P=0.0001)$. A significant quadratic relationship of seed viability $\left(R^{2}=0.510, P=0.0138\right)$ and germination $\left(R^{2}=0.768, P=0.0002\right)$ with the altitude of seed source was found. Seeds from populations between 3000 and $3350 \mathrm{msnm}$ (middle of the altitudinal distribution) had the highest viability (48.7\%) and germination (19.6\%), higher than those of the upper and lower altitudinal limits. If collecting is made at the intermediate altitude zone (zone II from 3000 to $3350 \mathrm{msnm}$ ), approximately $2.0 \mathrm{~kg}$ of seed will be required to produce 10,000 seedlings in the nursery; in contrast, the same goal would require five times more seed from the lower altitude zone $(11.1 \mathrm{~kg}$, Zone I, 2650 to $3000 \mathrm{msnm}$ ). This indicates the practical difficulties for supplying seed that a large-scale reforestation program would face, especially if germplasm originating from low-altitude populations is required, to implement assisted migration to higher altitudes to lessen the effects of climate change.

Index words: Abies religiosa, climate change, germination, altitudinal gradient, assisted migration, viability.

\section{INTRODUCCIÓN}

Abies religiosa (HBK) Schl. \& Cham. (oyamel) es una conífera nativa de las montañas del Eje Volcánico Transversal y la Sierra Madre del Sur de México (Rzedowski, 1978). Crece dentro de un intervalo altitudinal entre 2400 y 3600 msnm, en los estados de Hidalgo, Puebla, Veracruz, Michoacán, Jalisco, Morelos, Ciudad de México, Estado de México, Guerrero, Oaxaca y Tlaxcala (Sánchez-Velásquez et al., 1991). El bosque de oyamel que se ubica en la frontera del Estado de México y Michoacán, dentro de la Reserva de la Biósfera de la Mariposa Monarca (RBMM) funge como sitio de refugio y estancia invernal de las poblaciones de mariposa monarca Danaus plexippus L. (Anderson y Brower, 1996).

A pesar del esfuerzo y las prácticas de conservación dentro de la RBMM, existe preocupación por el futuro de Abies 
religiosa en la región, debido no sólo al cambio de uso de suelo (Champo-Jiménez et al., 2012; Vidal et al., 2014), sino porque como consecuencia del cambio climático se espera una reducción del nicho climático apropiado para su desarrollo. La reducción proyectada es de 69 \% para la década centrada en 2030, 87 \% para 2060 y $97 \%$ para 2090, de manera que para finales de siglo el nicho climático del oyamel ya no existiría dentro de la RBMM (Sáenz-Romero et al., 2012).

Abies religiosa presenta diferenciación morfológica (Castellanos-Acuña et al., 2014) y genética (Ortiz-Bibian et al., 2017; Graciano-Ávila et al., 2019) entre poblaciones a lo largo del gradiente altitudinal, lo cual es un indicador de la adaptación local a nivel de poblaciones (Rehfeldt, 1988). Esto es especialmente importante para las especies de distribución alpina, donde los efectos del cambio climático se intensifican (Lenoir et al., 2008). Las poblaciones arbóreas quedarán desfasadas del clima que les es propicio (Rehfeldt et al., 2012), ya que éste ocurrirá a cada vez mayores altitudes en las zonas montañosas de México (Graciano-Ávila et al., 2019; Sáenz-Romero et al., 2010); por ello, la continuidad de las poblaciones naturales dependerá de la capacidad de adaptación (la que depende a su vez de la estructura y diversidad genética poblacional y plasticidad fenotípica de la especie), de la velocidad y capacidad de migración de la especie, así como de las barreras que impidan la migración de las poblaciones (Greenwood et al., 2014; Lenoir et al., 2008; Palmroth et al., 1999; Pigliucci et al., 2006).

Las coníferas que presentan una historia de vida longeva, como A. religiosa, se verán limitadas, tanto para su dispersión en altitud como disponibilidad de tiempo, para adaptarse a la velocidad de los cambios climáticos, ya que A. religiosa alcanza su madurez sexual entre los 17 y 25 años de edad (Madrigal-Sánchez, 1964; Com. Pers.)' y es probable que posea una limitada capacidad de dispersión de sus semillas, no cuantificada para A. religiosa, pero en promedio $31 \mathrm{~m}$ para Abies alba Mill. (Cremer et al., 2012), lo que hace lento el proceso de migración natural.

Se ha sugerido realizar una migración asistida de las poblaciones de oyamel en la zona de estudio; ésto consistiría en recolectar semilla, producir planta en vivero y plantarla a una altitud mayor que la fuente de semillas, lo que significa desplazarse entre 275 (Sáenz-Romero et al., 2012) y 350 m (Ortiz-Bibian et al., 2017) a mayor altitud, lo que permitiría realinear las poblaciones al clima que les es propicio, pero que en el futuro ocurrirá a mayor altitud.

Para llevar a cabo lo anterior se requerirá una producción

'Madrigal-Sánchez X. (1964) Contribución al conocimiento de la ecología de bosques de oyamel Abies religiosa (H.B.K.), SCHL et CHAM.) en el valle de México. Tesis profesional. Escuela Nacional de Ciencias Biológicas, Instituto Politécnico Nacional. México, D. F. 111 p. masiva de planta en vivero, por lo que el presente trabajo tuvo como objetivos: 1) evaluar la viabilidad y capacidad germinativa de las semillas de $A$. religiosa procedente de 15 poblaciones de oyamel ubicadas a lo largo de un gradiente altitudinal en el Cerro de San Andrés, Michoacán, México; 2) identificar la existencia de diferenciación entre poblaciones en la capacidad germinativa y 3 ) determinar con mayor precisión la cantidad de semilla que sería necesario recolectar para la producción suficiente en número de plantas en vivero.

\section{MATERIALES Y MÉTODOS}

\section{Recolecta de semillas}

En la última semana de noviembre de 2010 se recolectaron conos maduros de 11 árboles al azar de cada una de 15 poblaciones de A. religiosa en el Cerro de San Andrés, Michoacán, México, en un área cercana a la Reserva de la Biósfera de la Mariposa Monarca (RBMM), en un gradiente altitudinal de 2850 a 3550 msnm; cada sitio se ubicó a $50 \mathrm{~m}$ de diferencia altitudinal con respecto al sitio de recolecta siguiente. La ubicación geográfica se muestra en la Figura 1 y las coordenadas y caracterización ecológica en el Cuadro 1. Los árboles recolectados se ubicaron a una distancia mínima de $30 \mathrm{~m}$ entre cada uno, con la finalidad de disminuir las posibilidades de coancestría. A cada sitio de recolección se le denominó procedencia y al conjunto de árboles muestreados y representados en el experimento por semillas y plántulas se le denominó población.

\section{Caracterización ecológica}

Se realizó en sitios circulares de $1000 \mathrm{~m}^{2}$, un sitio por cada nivel de altitud; en ellos se registró el diámetro a la altura del pecho (DAP, con forcípula) y la altura con clinómetro (Marca Suunto, Vantaa, Finland) de las especies arbóreas presentes. A partir del DAP se estimó el área basal. Los árboles se agruparon en tres categorías: A. religiosa, Pinus sp. y hojosas (todos los árboles diferentes a las coníferas).

\section{Manejo de la semilla}

Los conos fueron secados durante $19 \mathrm{~d}$ en un invernadero de la Unidad San Juanito Itzícuaro del Instituto de Investigaciones Agropecuarias y Forestales de la Universidad Michoacana de San Nicolás de Hidalgo (UMSNH), en Morelia, Michoacán. Una vez secos los conos, las semillas se extrajeron manualmente, se mezcló la semilla por procedencia y se utilizó una cantidad equitativa de semillas de cada individuo. Debido a que las semillas de esta especie, una vez extraídas, presentan microgotas de resina en su exterior, la separación de semillas vanas y vacías 


\section{Cerro de San Andrés}

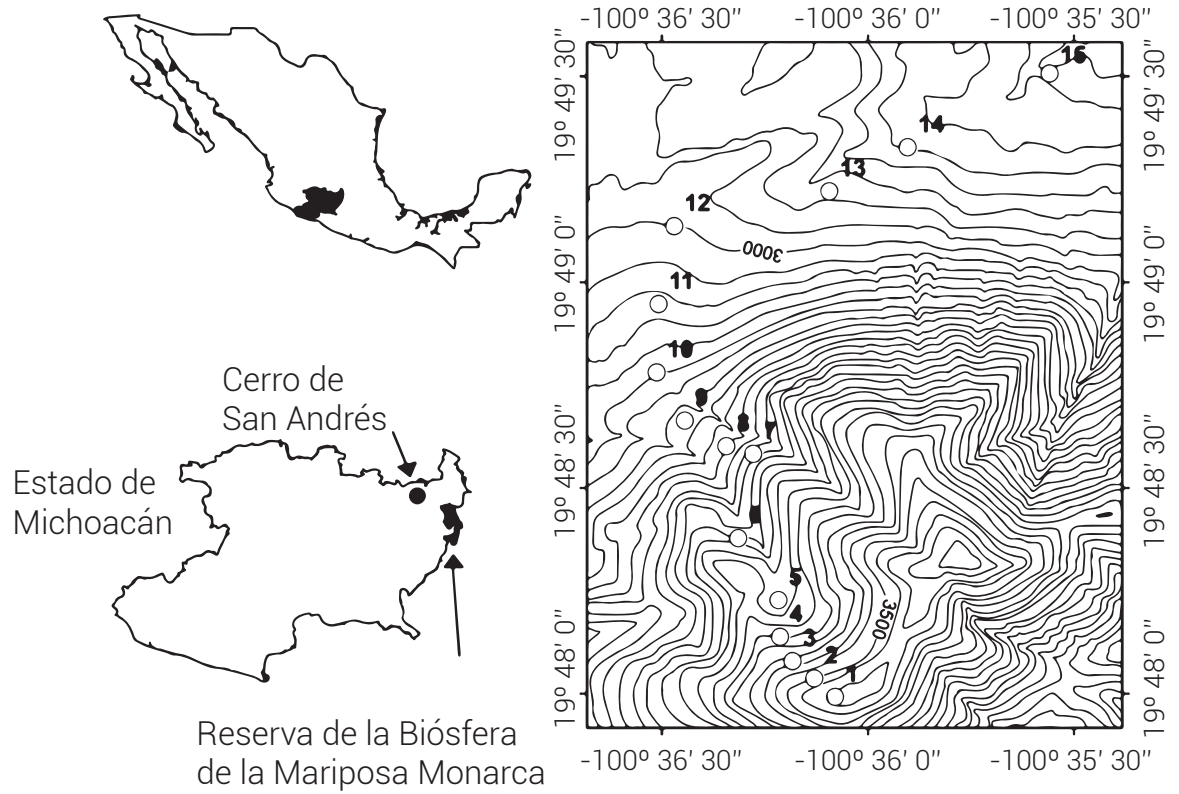

Figura 1. Localización geográfica de las 15 poblaciones de Abies religiosa colectadas en el cerro de San Andrés, Michoacán, México.

resulta sumamente difícil, ya que las semillas se adhieren fácilmente entre sí, lo que dificulta tal separación mediante técnicas comunes, como la exposición a una corriente de viento o una columna de aire en un dispositivo de laboratorio; por ello, no se eliminaron las semillas vanas en esta etapa.

\section{Pruebas de viabilidad y germinación}

De cada una de las 15 poblaciones se tomaron 200 semillas al azar a las que se realizó una prueba de viabilidad mediante la prueba de tetrazolio (cloruro de 2,3,5-trifeniltetrazolio) (Sigma-Aldrich $®$ ) siguiendo la metodología descrita por la ISTA (2006). La muestra se dividió en cuatro repeticiones de 50 semillas cada una. A cada semilla se le efectuó un pequeño corte en el extremo contrario a donde emerge la radícula para facilitar el permeado del reactivo y se sumergieron en una solución de cloruro de 2, 3, 5-trifenil tetrazolio $\left(0.8 \mathrm{mg} 100 \mathrm{~mL}^{-1}\right.$ de agua destilada) en la obscuridad. Después de $24 \mathrm{~h}$, las semillas se sacaron de la solución y se realizó un corte transversal en cada una de ellas; si el embrión se teñía de color púrpura indicaba que el embrión era viable. El total de semillas examinadas se ajustó excluyendo las semillas que, al realizar el corte, se encontraban vacías.

Para el ensayo de germinación se utilizaron 400 semillas de cada población. Las 400 semillas se dividieron en ocho grupos de 50 semillas; cada grupo se colocó en una caja de Petri con papel filtro y se incubaron por un periodo de 28 d en una cámara de crecimiento (Lumistell ${ }^{\circledR}$, Modelo ICP18, Celaya, Guanajuato, México), con ciclos de 12 h luz/12 h obscuridad, a una temperatura de $25^{\circ} \mathrm{C}$. Las semillas se humedecieron cada $3 \mathrm{~d}$ con agua destilada con Captán ${ }^{\circledR}$ a una concentración de $0.8 \mathrm{~g} 100 \mathrm{~mL}^{-1}$. El criterio usado para determinar la germinación fue la emergencia de la radícula; los datos de germinación se tomaron cada tercer día.

Se determinó el peso de la semilla; para ello se tomaron al azar 400 semillas de cada una de las 15 poblaciones, se agruparon en ocho repeticiones de 50 semillas y se pesaron en una balanza electrónica con precisión de $0.001 \mathrm{~g}$ (Ohaus SP402, Parsippany, NJ, USA), a fin de facilitar la estimación de la cantidad de semilla necesaria para producción de planta en vivero, tras considerar la capacidad germinativa.

\section{Análisis estadístico}

Para determinar diferencias estadísticas entre poblaciones en viabilidad, germinación y peso de semilla se realizó un análisis de varianza mediante el procedimiento GLM de SAS (SAS Institute, 2004).

$$
Y_{i j k}=\mu+B_{i}+P_{j}+\varepsilon_{i j k}
$$

donde: $\mu$ es la media general, $B_{i}$ el efecto del $i$-ésimo bloque, $P_{j}$ el efecto de la j-ésima procedencia y $\varepsilon_{i j k}$ es el error. Las procedencias se consideraron como un factor de efectos fijos, en cuanto a que la intención fue comparar los valores promedio de las poblaciones y determinar diferencias estadísticas entre ellas. 


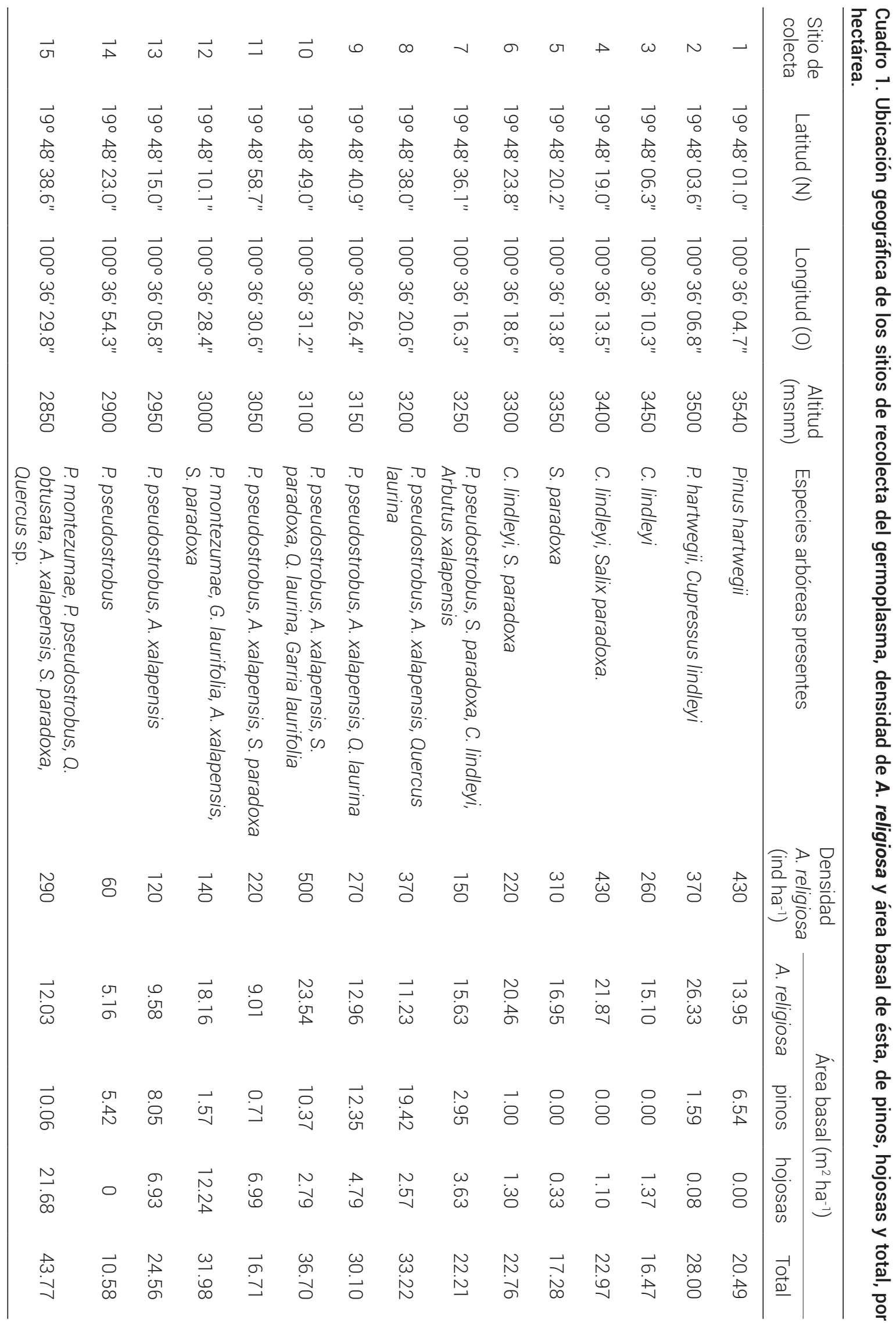


Para determinar el patrón altitudinal de variación de viabilidad y capacidad germinativa se realizó un análisis de regresión (lineal y cuadrático) de los valores medios de las poblaciones $\left(Y_{i}\right)$ con respecto a su altitud $\left(X_{i}\right)$, mediante el procedimiento REG de SAS (SAS Institute, 2004) con los siguientes modelos estadísticos:

$$
\begin{gathered}
Y_{i}=\beta_{0}+\beta_{7} X_{i}+\varepsilon_{i} \\
Y_{i}=\beta_{0}+\beta_{7} X_{i}+\beta_{2} X_{i}^{2}+\varepsilon_{i}
\end{gathered}
$$

donde: $Y_{i}$ es valor medio de la $i$-ésima procedencia, $\beta_{0}$ es el intercepto, $\beta_{1}$ y $\beta_{2}$ son los parámetros de regresión, $X_{i}$ es la altitud de la $i$-ésima procedencia y $\varepsilon_{i}$ el error aleatorio.

Se realizó una estimación de la cantidad de semilla $(\mathrm{kg})$ necesaria para obtener 10,000 plántulas en vivero como promedio de poblaciones agrupadas en zonas altitudinales de acuerdo con la zonificación basada en características morfológicas (Castellanos-Acuña et al., 2014); se utilizó la fórmula reportada por la FAO (1991):

$$
Y=10000 /(\text { S G F) }
$$

donde: $Y$ es el número de $\mathrm{kg}$ a colectar para producir aproximadamente 10,000 plantas en vivero; $S$ es el número promedio de semillas por kg en las poblaciones de una misma zona altitudinal; $G$ es el porcentaje promedio de germinación en las poblaciones de una misma zona altitudinal, expresado como proporción; F es un factor de corrección (0.85), que considera un $15 \%$ de mortalidad de planta en vivero, con base en experiencia previa.

\section{RESULTADOS Y DISCUSIÓN}

\section{Viabilidad y germinación de la semilla}

En el análisis de varianza de la viabilidad de la semilla se encontraron diferencias significativas entre poblaciones ( $P$ $\leq 0.0001)$. En la regresión entre la viabilidad promedio por población y la altitud de origen de la población, se observó que las poblaciones de la parte central de la distribución altitudinal (de 3100 a 3400 msnm) tienen un mayor número de semillas viables (hasta $57 \%$ ) que las de los extremos altitudinales superior e inferior (Figura 2); este patrón es estadísticamente significativo bajo el modelo de regresión cuadrático $\left(R^{2}=0.510, P \leq 0.01\right)$.

Los resultados de germinación fueron similares a los de viabilidad en cuanto a la tendencia de los datos entre procedencias. Se encontraron diferencias significativas entre poblaciones en el porcentaje de germinación $(P \leq$ 0.0001). El promedio general de germinación fue bajo (13 $\%$ ). Debe considerarse que este valor muy probablemente está subestimado, ya que, por las dificultades explicadas en metodología, no se eliminaron las semillas vanas antes de hacer la prueba de germinación.

La germinación promedio por población presenta un pronunciado patrón altitudinal bajo un modelo cuadrático $\left(R^{2}=0.768, P=0.0002\right)$, donde las poblaciones de la parte central de la distribución altitudinal (de 3100 a 3400 m de altitud) producen semillas con mayor porcentaje de germinación ( $20 \%$ en promedio, con valores máximos de $27 \%)$ que las poblaciones de los extremos altitudinales

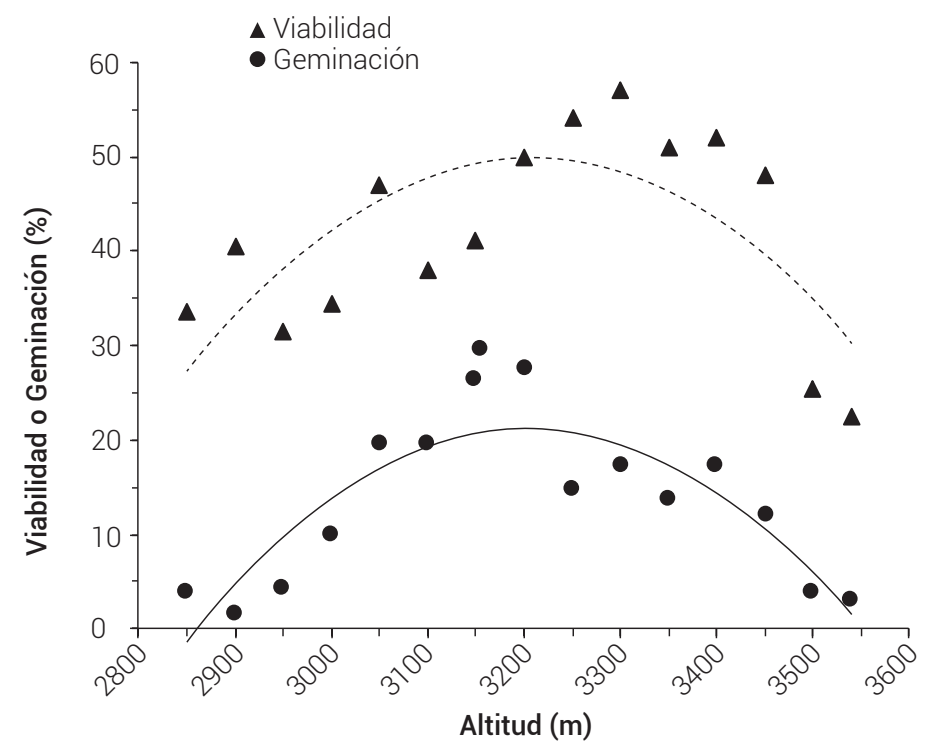

Figura 2. Porcentaje de viabilidad $\left(R^{2}=0.510 ; P=0.0138\right)$ y germinación $\left(R^{2}=0.7680 ; P=0.0002\right)$ promedio de la semilla por población de Abies religiosa en relación con la altitud de origen. 
superior (3400 a 3550 msnm) e inferior (2850 a 3000 msnm), con un porcentaje muy bajo, de 9 y $5 \%$ de germinación promedio, respectivamente (Figura 2).

Las curvas de regresión de los porcentajes de viabilidad y de germinación son similares (Figura 2); esto indica que aun cuando la germinación está probablemente subestimada debido a que no se eliminaron las semillas vanas en la prueba, la estimación de la tendencia de la capacidad germinativa parece ser robusta; las procedencias de la parte central de la distribución altitudinal tienen más capacidad germinativa que las de los extremos altitudinales.

El peso promedio de semillas por kg también tuvo un patrón similar al de viabilidad y germinación; existieron diferencias significativas entre procedencias ( $P \leq$ 0.0001), con un pronunciado patrón altitudinal, donde las procedencias de los extremos altitudinales tuvieron un menor peso que las de la parte central de la distribución altitudinal (modelo cuadrático, $R^{2}=0.757, P=0.0008$, que excluye dos poblaciones atípicas).

El pronunciado patrón altitudinal encontrado podría deberse a que los árboles de las procedencias de los extremos altitudinales se encuentran expuestos a condiciones ambientales extremas. Las poblaciones de mayor altitud, entre 3500 y 3550 msnm, estarían expuestas a la ocurrencia frecuente de heladas, mientras que las del extremo altitudinal inferior probablemente lo están a estrés por sequía, ya que conforman el "límite xérico" (Mátyás, 2010). Probablemente el estrés ambiental en los límites altitudinales dificulta los procesos de formación de la semilla, tales como la polinización, fertilización y translocación de reservas para el llenado de la misma, como parece ocurrir con Pinus pseudostrobus (López-Toledo et al., 2017). El papel que juegan las condiciones ambientales en la fenología de las plantas ha sido ya reportado (Vitasse et al., 2009).

Si bien estudios de germinación realizados con Pseudotsuga menziesii (Mirb.) Franco en zonas boscosas fragmentadas y con poblaciones aisladas, reportan un indiscutible detrimento en el porcentaje de germinación de las semillas debido a la baja densidad del arbolado (MápulaLarreta et al., 2008), en este caso la densidad de A. religiosa no parece haber influido de manera decisiva en el patrón altitudinal encontrado. No se encontró una asociación significativa entre la media por población de la viabilidad de las semillas de A. religiosa y las características del rodal, tales como densidad de individuos por hectárea (modelo lineal, $R^{2}=0.020, P=0.6125$ ) o área basal (modelo lineal, $\left.R^{2}=0.007, P=0.7600\right)$; tampoco se encontró asociación entre la germinación y la densidad de individuos $\left(\mathrm{R}^{2}=\right.$ $0.080, P=0.3068)$ o área basal $\left(R^{2}=0.093, P=0.2694\right)$; sin embargo, estos datos deben tomarse con cautela, ya que la caracterización de los rodales se realizó tres años después de la recolecta de semillas y se encontraron evidencias de perturbación debido a tala, particularmente en sitios de baja altitud; por ello, la densidad de individuos y su área basal podría estar subestimada.

Los bajos niveles de viabilidad y germinación encontrados son congruentes con los reportados en otros estudios; por ejemplo, Nieto de Pascual-Pola et al. (2003) reportaron $37 \%$ de viabilidad en semillas de A. religiosa de Río Frío, Ixtapaluca, México. En otro estudio realizado hace más de 30 años también se encontró un bajo porcentaje de germinación, de entre 45 y 49 \% para esta especie, sin aplicar ningún tratamiento pre-germinativo y con semilla recién recolectada (Patiño-Valera et al., 1983).

Los bajos niveles de viabilidad y germinación también se han reportado para otras especies de Abies. En A. guatemalensis se encontró una germinación promedio de sólo 15 \% (INAB, 2017); si bien en ese estudio no se realizaron pruebas de viabilidad, necesariamente el porcentaje de semillas viables es mayor o igual al de germinación. Arista (1993) reportó 26 \% de germinación en semillas de Abies pinsapo Boiss. en el Parque Natural Sierra de Grazalema, España. Al menos en el caso de las especies de Abies en México, ésto podría estar relacionado con que las poblaciones en general presentan un exceso de homocigotos, lo que indica altos niveles de endogamia, según análisis de isoenzimas (Eguiarte-Fruns y Furnier, 1997; Com. Pers.)2.

En cuanto a la velocidad de germinación, a los 7 d de que se inició el ensayo de germinación se registraron las primeras semillas germinadas. Las procedencias alcanzaron la mayor germinación acumulada al día 14; posteriormente, la germinación decreció de manera importante y después del día 21 ya no hubo germinación de semillas. Los datos de velocidad de germinación aquí obtenidos son en gran medida coincidentes con los de Mayen (1987; Com. Pers.) ${ }^{3}$, quien indica que el mayor porcentaje de semillas de Abies religiosa germina a los 12 d. De acuerdo con los resultados de este trabajo, entre los días 12 y 14 se encontró la germinación máxima.

\section{Clasificación de la calidad de semilla por zona altitudinal para su uso en vivero}

Utilizando los límites definidos de zonas altitudinales para A. religiosa en la región estudiada con base en la

${ }^{2}$ Eguiarte-Fruns L. E. y G. R. Furnier (1997) Niveles y patrones de variación genética del género Abies en México. Informe Final SNIBCONABIO Proyecto No. B138. Instituto de Ecología, Universidad Nacional Autónoma de México. México, D. F. 50 p.

${ }^{3}$ Mayen L. (1987) Evaluación de los efectos de tres tipos de agua en el tiempo y porciento de germinación de semillas de oyamel (Abies religiosa). Tesis profesional. FES-Cuautitlán, UNAM. Cuautitlán Izcalli, Edo. de México. 
diferenciación genética entre poblaciones (Ortiz-Bibian et al., 2017) es posible agrupar las poblaciones por zona altitudinal y estimar un promedio del número de $\mathrm{kg}$ de semilla necesarios para producir plantas en vivero. Esto permite simplificar el trabajo de planeación del esfuerzo necesario para colectar semilla para abastecer programas de reforestación a gran escala (Alía et al., 2005).

Para producir 10,000 plantas en vivero, si la semilla se recolecta de la zona de altitud intermedia (3000 a 3350 msnm) se requieren aproximadamente $2.0 \mathrm{~kg}$; en cambio, la misma meta de producción en vivero requiere tres veces más semilla de la zona de mayor altitud $(6.1 \mathrm{~kg}, 3350 \mathrm{a}$ $3700 \mathrm{msnm}$ ) y casi cinco veces más semilla de la zona de menor altitud (11.1kg, 2650 a 3000 msnm) (Cuadro 2).

La gran diferencia en semilla necesaria por cada zona altitudinal se debe principalmente a las diferencias en capacidad germinativa de las poblaciones, mas que al promedio del número de semillas por $\mathrm{kg}$, que es muy semejante entre zonas, entre 32,708 y 33,916 semillas kg-1 (Cuadro 2) y relativamente cercana a la señalada en la ficha técnica de la especie (CONAFOR, 2013), de 26,599 semillas por kg y a los valores obtenidos por Nieto de Pascual-Pola et al. (2003), de 23,054 semillas por kg.

El resultado anterior indica las dificultades a las que se enfrentaría un programa de reforestación que contemple como un factor relevante el origen altitudinal de la semilla tras considerar o no el cambio climático. Si se considera únicamente que existe diferenciación genética entre poblaciones se podría reforestar usando semilla de la misma zona altitudinal que el sitio de plantación. Si adicionalmente a lo anterior se consideran los efectos del cambio climático sería necesario recolectar semilla en una zona altitudinal, producir planta en vivero y reforestar la zona altitudinal inmediata superior (Ortiz-Bibian et al., 2017). En ambos casos, la baja germinación de la semilla de la zona altitudinal inferior representará un reto para lograr el abasto de semilla a los viveros.

\section{Limitaciones de este estudio}

La cuantificación de semillas vanas y su eliminación de cada lote de semillas por procedencia previo a la germinación, indudablemente, habría sido de gran utilidad para explicar las diferencias en capacidad germinativa a lo largo del gradiente altitudinal. Tal análisis se recomienda para futuros trabajos a pesar de la dificultad que ocasiona la presencia de resina en los conos y semillas de esta especie.

\section{CONCLUSIONES}

Las poblaciones que se encuentran en la parte media del gradiente altitudinal de Abies religiosa en la región de la Reserva de la Biósfera de la Mariposa Monarca poseen el promedio más alto de viabilidad y de germinación de la semilla, en comparación con las poblaciones de los extremos altitudinales. Si se recolecta de la altitud intermedia (de 3000 a 3350 msnm) se requieren aproximadamente $2.0 \mathrm{~kg}$ de semilla para producir 10,000 plantas en vivero; en cambio, la misma meta requiere de casi cinco veces más semilla de la zona de menor altitud (2650 a 3000 m). Esto indica las dificultades que enfrentaría un programa de reforestación que contemple como un factor relevante el origen altitudinal de la semilla, ya que la baja capacidad germinativa de la semilla, especialmente de la zona altitudinal inferior, representa un reto para lograr el abasto de semilla a los viveros.

\section{AGRADECIMIENTOS}

Se agradece el financiamiento del Monarch Butterfly Fund, del Fondo Mexicano para la Conservación de la Naturaleza apoyado por el Servicio Forestal de los Estados Unidos (USFS) y la Agencia de los Estados Unidos para el Desarrollo Internacional (USAID) en el marco del Grupo de Trabajo sobre Recursos Genéticos Forestales de la Comisión Forestal de América del Norte, del Fondo Mixto CONACYT-Michoacán (FOMIX-2009-127128), de la Coordinación de la Investigación Científica de la UMSNH

Cuadro 2. Cantidad de semilla necesaria por zona altitudinal para producir 10,000 plantas en vivero, en función del número de semillas $\mathrm{kg}^{-1}$, el porcentaje promedio de germinación y una pérdida en vivero estimada en $15 \%$.

\begin{tabular}{|c|c|c|c|c|}
\hline Zona $^{+}$ & $\begin{array}{l}\text { Intervalo altitudinal } \\
\text { (msnm) }\end{array}$ & $\begin{array}{c}\text { Germinación promedio } \\
(\%)\end{array}$ & Número de semillas $\mathrm{kg}^{-1}$ & $\begin{array}{c}\text { kg de semilla a recolectar } \\
\text { para producir } 10,000 \\
\text { plántulas }\end{array}$ \\
\hline । & $2650-3000$ & 9.0 & 33,916 & 6.1 \\
\hline ॥ & $3000-3350$ & 19.9 & 32,114 & 2.0 \\
\hline III & $3350-3700$ & 4.9 & 32,708 & 11.1 \\
\hline
\end{tabular}

†Zonificación altitudinal según Ortiz-Bibian et al. (2017). 
y del Programa PAPIIT (IN202112) de la UNAM. Se agradece al Sr. Gabriel Muñoz Montoya (Queréndaro, Michoacán) por las facilidades otorgadas para la recolecta. A Consuelo Marín-Togo por la preparación del mapa. Comentarios en la preparación de la versión original de Arnulfo Blanco-García e Yvonne Herrerías-Diego, Facultad de Biología de la UMSNH, y posteriormente un editor asignado y dos revisores anónimos ayudaron a mejorar sustancialmente el manuscrito.

\section{BIBLIOGRAFÍA}

Alía M. R., N. Alba M., D. Agúndez L. y S. Iglesias S. (2005) Manual para la Comercialización y Producción de Semillas y Plantas Forestales. Materiales de Base y de Reproducción. Serie Forestal. Dirección General para la Biodiversidad. Madrid. 384 p.

Anderson J. B. and L. P. Brower (1996) Freeze-protection of overwintering monarch butterflies in Mexico: critical role of the forest as a blanket and umbrella. Ecological Entomology 21:107-116, https://doi.org/10.1111/j.1365-2311.1996.tb01177.x

Arista M. (1993) Germinación de las semillas y supervivencia de las plántulas de Abies pinsapo Boiss. Acta Botánica Malacitana 18:173-177.

Castellanos-Acuña D., R. A. Lindig-Cisneros, M. A. Silva-Farías y C. SáenzRomero (2014) Zonificación altitudinal provisional de Abies religiosa en un área cercana a la Reserva de la Biósfera de la Mariposa Monarca, Michoacán. Revista Chapingo Serie Ciencias Forestales y del Ambiente 20:215-225, https://doi.org/10.5154/r.rchscfa.2013.11.041

Champo-Jiménez O., L. Valderrama-Landeros y M. L. España-Boquera (2012) Pérdida de cobertura forestal en la reserva de la biosfera Mariposa Monarca, Michoacán, México (2006-2010). Revista Chapingo Serie Ciencias Forestales y del Ambiente 18:143-157, https://doi.org/10.5154/r.rchscfa.2010.09.074

CONAFOR, Comisión Nacional Forestal (2013) Abies religiosa (Kunt Schltdl. et Cham.). Paquetes Tecnológicos. CONAFOR, CONABIO, SIRE. México, D. F. http://www.conafor.gob. mx:8080/documentos/docs/13/873Abies\%20religiosa.pdf (Diciembre 2013)

Cremer E., B. Ziegenhagen, K. Schulerowitz, C. Mengel, K. Donges, R. Bialozyt, E. Hussendörfer and S. Liepelt (2012) Local seed dispersal in European silver fir (Abies alba Mill.): lessons learned from a seed trap experiment. Trees 26:987-996, https://doi.org/10.1007/s00468-012-0676-9

FAO, Organización de la Naciones Unidas para la Agricultura y la Alimentación (1991) Guía para la Manipulación de Semillas Forestales. FAO y Centro de Semillas Forestales de DANIDA. Roma, Italia. http://www.fao.org/3/ad232s/ad232s00.htm (Diciembre 2013)

Graciano-Ávila G., E. Alanís-Rodríguez, 0. A. Aguirre-Calderón, M. A. González-Tagle, E. J. Treviño-Garza, A. Mora-Olivo y E. BuendíaRodríguez (2019) Estimación de volumen, biomasa y contenido de carbono en un bosque de clima templado-frío de Durango, México. Revista Fitotecnia Mexicana 42:119-127.

Greenwood S., J. C. Chen, C. T. Chen and A. S. Jump (2014) Strong topographic sheltering effects lead to spatially complex treeline advance and increased forest density in a subtropical mountain region. Global Change Biology 20:3756-3766, https://doi.org/10.1111/gcb.12710

INAB, Instituto Nacional de Bosques (2017) Pinabete (Abies guatemalensis Rehder). Paquete Tecnológico Forestal. INAB. Guatemala, Guatemala. 40 p.

ISTA, International Seed Testing Association (2006) International Rules for Seed Testing. International Seed Testing Association. Zurich, Switzerland. $333 p$

Lenoir J., J. C. Gégout, P. A. Marquet, P. de Ruffray and H. Brisse (2008) A significant upward shift in plant species optimum elevation during the 20th Century. Science 320:1768-
1771, https://doi.org/10.1126/science.1156831

López-Toledo L., M. Heredia-Hernandez, D. Castellanos-Acuña, A. BlancoGarcía and C. Sáenz-Romero (2017) Reproductive investment of Pinus pseudostrobus along an altitudinal gradient in Western Mexico: implications of climate change. New Forests 48:867881, https://doi.org/10.1007/s11056-017-9602-8

Mápula-Larreta M., J. López-Upton, J. J. Vargas-Hernández and A. Hernández-Livera (2008) Germinación y vigor de semillas en Pseudotsuga menziesii de México. Ra Ximhai 4:119-134, https://doi.org/10.35197/rx.04.01.2008.07.mm

Mátyás C. (2010) Forecasts needed for retreating forests. Nature 464:1271, https://doi.org/10.1038/4641271a

Nieto de Pascual-Pola C., M. Á. Musálem y J. Ortega-Alcalá (2003) Estudio de algunas características de conos y semillas de Abies religiosa (HBK) Schl. et Cham. Agrociencia 37:521-531.

Ortiz-Bibian M. A., A. Blanco-García, R. A. Lindig-Cisneros, M. GómezRomero, D. Castellanos-Acuña, Y. Herrerías-Diego, N. M. SánchezVargas and C. Sáenz-Romero (2017) Genetic variation in Abies religiosa for quantitative traits and delineation of elevational and climatic zoning for maintaining Monarch Butterfly overwintering sites in Mexico, considering climatic change. Silvae Genetica 66:14-23, https://doi.org/10.1515/sg-2017-0003

Palmroth S., F. Berninger, E. Nikinmaa, J. Lloyd, P. Pulkkinen and P. Hari (1999) Structural adaptation rather than water conservation was observed in Scots pine over a range of wet to dry climates. Oecologia 121:302-309, https://doi.org/10.1007/s004420050932

Patiño-Valera F., P. De la Garza, Y. Villagómez A., I. Talavera A. y F. Camacho M. (1983) Guía para la recolección y manejo de semillas de especies forestales. Boletín de Divulgación No. 63. Instituto Nacional de Investigaciones Forestales, Secretaría de Agricultura y Recursos Hidráulicos. México, D.F. $181 \mathrm{p}$

Pigliucci M., C. J. Murren and C. D. Schlichting (2006) Phenotypic plasticity and evolution by genetic assimilation. Journal of Experimental Biology 209:2362-2367, https://doi.org/10.1242/jeb.02070

Rehfeldt G. E. (1988) Ecological genetics of Pinus contorta from the Rocky Mountains (USA): a synthesis. Silvae Genetica 37:131-135.

Rehfeldt G. E., N. L. Crookston, C. Sáenz-Romero and E. M. Campbell (2012) North American vegetation model for land-use planning in a changing-climate: a solution to large classification problems. Ecological Applications 22:119141, https://doi.org/10.1890/11-0495.1

Rzedowski J. (1978) Vegetación de México. Limusa. México, D. F. 432 p.

Sáenz-Romero C., G. E. Rehfeldt, N. L. Crookston, P. Duval, R. StAmant, J. Beaulieu and B. A. Richardson (2010) Spline models of contemporary, 2030, 2060 and 2090 climates for Mexico and their use in understanding climatechange impacts on the vegetation. Climatic Change 102:595-623, https://doi.org/10.1007/s10584-009-9753-5

Sáenz-Romero C., G. E. Rehfeldt, P. Duval and R. A. Lindig-Cisneros (2012) Abies religiosa habitat prediction in climatic change scenarios and implications for monarch butterfly conservation in Mexico. Forest Ecology and Management 275:98-106, https://doi.org/10.1016/j.foreco.2012.03.004

Sánchez-Velásquez L. R., M. R. Pineda-López y A. Hernández-Martínez (1991) Distribución y estructura de la población de Abies religiosa (H.B.K.) Schl. et Cham., en el Cofre de Perote, estado de Veracruz, México. Acta Botánica Mexicana 16:45-55, https://doi.org/10.21829/abm16.1991.625

SAS Institute (2004) SAS/STAT® 9.1 User's Guide. 3rd edition. SAS Institute Inc. Cary, North Carolina, USA. 5121 p.

Vidal 0., J. López-García and E. Rendón-Salinas (2014) Trends in deforestation and forest degradation after a decade of monitoring in the Monarch Butterfly Biosphere Reserve in Mexico. Conservation Biology 28:177-186, https://doi. org/10.1111/cobi.12138

Vitasse Y., S. Delzon, C. C. Bresson, R. Michalet and A. Kremer (2009) Altitudinal differentiation in growth and phenology among populations of temperate-zone tree species growing in a common garden. Canadian Journal of Forest Research 39:1259-1269, https://doi.org/10.1139/X09-054 